\title{
The twisted role of natalizumab and rehabilitation in an aggressive form of multiple sclerosis: a case report
}

\author{
CORDOS M. Adrian ${ }^{1}$, BARAC I. Simina ${ }^{2}$, MUREȘANU F. Dafin ${ }^{2}$
}

Corresponding author: Barac I. Simina, E-mail: siminabarac@gmail.com

DOI: http://dx.doi.org/10.12680/balneo.2020.409 Vol.11, No.4, December 2020

1 County Emergency Hospital Cluj-Napoca, Neurology Clinic, Romania, Victor Babeș street, number 43, Cluj-Napoca, Romania 2 Department of Neurosciences, "Iuliu Haţieganu" University of Medicine and Pharmacology Cluj-Napoca, Romania

\begin{abstract}
Multiple sclerosis is one of the main causes of neurological disability among young people, severely influencing life's quality. Motor deficit is one of the most invalidating symptoms in multiple sclerosis. Motor involvement at onset, spinal demyelinating lesion distribution, highly active lesions, high frequency of relapses in the first 2 years after disease onset and post relapse residual disability with poor recovery, later age at onset and male gender represent unfavorable prognostic factors. Considering the significant role of prognostic factors in predicting the evolution of the disease, an induction treatment approach should always be considered. In this report we present the case of a middle-aged male patient presenting severe weakness and sensitive symptoms with a challenging therapeutic decision that had a favorable recovery after reconsidering the initial therapy. The recognition of aggressive forms of multiple sclerosis is mandatory in preventing further disability, improving the patient's quality of life. Natalizumab is a monoclonal antibody used for the aggressive forms of multiple sclerosis, reducing the lymphocytes traffic through the blood brain barrier, with a great impact on relapses frequency and disease evolution.
\end{abstract}

Keywords: aggressive multiple sclerosis, brain derived neurotrophic factor, natalizumab, rehabilitation,

\section{Introduction}

Multiple sclerosis (MS) is an autoimmune, demyelinating, multi-focal disorder of the central nervous system (CNS) characterized by neuroinflammation and neurodegeneration (1). The activation of T lymphocytes plays the central role in demyelination, oligodendrocyte death and axonal loss (2). The etiology of MS is not completely elucidated, but a few environmental and genetic factors were identified as contributors to MS susceptibility. The environmental factors contributing to immune dysregulation in MS are: systemic viral infections (e.g. Epstein Barr virus), smoking, industrial chemicals, obesity, intestinal microbiome dysregulation and vitamin $\mathrm{D}$ deficiency $(3,4)$. The environmental factors maintain a peripheral pro-inflammatory status with elevated levels of circulating cytokines (5). There is also a strong evidence for genetic risk factors involved in MS susceptibility, supported by familial clustering. Studies evaluating the genetic background of the siblings of MS patients showed a 10-15 times higher risk of developing MS compared with general population (6). Recent large genome wide association studies (GWAS) suggested a strong association between human leukocyte antigen (HLA) class II region of the major histocompatibility complex (MHC) located in chromosome $6 \mathrm{p} 21$ and MS (6). The presence of allele DRB1*15:01 was correlated with younger age of MS onset (6).

In the pathogenetic process of MS, T lymphocytes, represented by CD4 (+) $\mathrm{T}$ cells are exposed to myelin antigens in the deep cervical lymph nodes. At this point, CD4 (+) $\mathrm{T}$ cells are activated, escaping the thymic mechanism of self-tolerance control and becoming effector cells once released in the peripheral blood stream (7). Gaining a migratory phenotype, CD4 (+) T cells, pass the modified blood-brain barrier (BBB), and blood-cerebrospinal fluid barrier (BCSFB), at the interface of choroid plexus and leptomeningeal vessels, entering the brain parenchyma (8). The main immunological battle field in the brain parenchyma is the perivenular space, where a second reactivation of CD4 $(+) \mathrm{T}$ cells occurs, in the presence of local antigen presenting cells (APCs) (7). The contact between CD4 $(+) \mathrm{T}$ cells and APC results in an elevated production of cytokines which triggers the leukocytes traffic from the blood stream through the BBB, enhancing the myelin damage (9). The resolution of the inflammatory process is mediated by the releasing of the brain-derived neurotrophic factors stimulating neuroplasticity and neuronal cells differentiation $(10,11)$.

The list of medical conditions that can cause multi-focal neurologic problems in young people is quite extensive and so the differential diagnosis of MS is far ranging. The diagnosis of multiple sclerosis is based on clinical, biological and imaging findings. The McDonald criteria incorporate all of these findings to confirm a definite diagnosis of MS, and have undergone multiple revisions in 2005, 2010, 2017 (12). Once a diagnosis of MS has been established, the therapeutic management may prove even more difficult, given the unpredictable evolution of the disease and the variety of drugs available, each with variable efficacy and considerable toxicity (13). At this point, there are two therapeutic approaches: an escalation approach or an induction approach with more aggressive therapies.

The clinical evolution of MS is highly dependent on the sequence of the immunological events.

The aggressive course of the disease is characterized by rapid disability accumulation (14). Several studies emphasized the role of early diagnosis in the aggressive forms of MS and the concept of a "therapeutic window of opportunity", gaining control over the diseases course and ameliorating the long term evolution (14). In order to control inflammation and 
stabilize the clinical evolution, a more aggressive treatment is needed. In our case report, we present the favorable clinical outcome of a patient with an aggressive form of MS under treatment with Natalizumab. Natalizumab is the only treatment directed against the $\alpha 4$ subunit of integrins. The $\alpha 4$ subunit of integrins expressed on the surface of $\mathrm{T}$ cells, attach to the vascular endothelial receptors and facilitate the adhesion of $\mathrm{T}$ cells to the vessels walls (10). The benefits of Natalizumab treatment are not restricted only to the reduction of $\mathrm{T}$ cells adhesion and traffic, but are also associated with an increased production of brain-derived neurotrophic factor (BDNF) involved in remyelination, neuroprotection and disease stabilization (10).

\section{Case presentation}

A 45-year old Caucasian male, residing in an urban area, with right laterality, was admitted to our Neurology department with complaints of numbness, paresthesia and weakness of the lower limbs associated with gait difficulty, symptoms which worsened during the last three weeks prior to admission.

The patient's history revealed that the onset of the disease was characterized by lower limbs paresthesia 5 years prior to admission with an insidiously, ascending and progressive pattern over time. He also associated progressive lower limbs weakness and gait difficulties. It is important to note that one year before the symptoms began to show the patient had been bitten by a tick and had developed a local erythematous reaction around the site of the bite. Extensive blood tests were performed at that time that revealed a positive IgG test for B. burgdorferi antibodies using the Western Blot analysis. A lumbar puncture was also performed and the CSF analysis revealed mild lymphocytic pleocytosis $\left(12 / \mathrm{mm}^{3}\right.$, 95\% lymphocytes), an elevated $\mathrm{IgG}$ index and a positive result for oligoclonal bands with normal protein levels. CSF anti- $B$. burgdorferi antibodies were absent. Contrast MRI scans of the brain and spinal cord were performed which found multiple demyelinating periventricular, subcortical and juxtacortical lesions (one of which was contrast-enhancing), and also multiple demyelinating lesions in the cervical and dorsal spinal cord (two of which were contrast-enhancing in the cervical region). Electroneurography and motor evoked potentials study were performed and the results were within normal range. A differential diagnosis between multiple sclerosis and Lyme disease with neuroborreliosis was thus proposed. The patient was given pulse IV methylprednisolone therapy and Ceftriaxone $(2 \mathrm{~g}$ per day for 30 days). During the course of antibiotherapy the motor deficit and paresthesia partially subsided but progressively worsened at the end of the therapy. Two more courses of antibiotherapy were given without significant benefits. In the following years the disease had an undulating course with two more attacks consisting of left hemiparesis and paresthesia regarding the upper limbs. MRI scans of the brain and spinal cord were also repeated, revealing new, contrast-enhancing, lesions in the brain and the cervical spinal cord (Figure 1). The reevaluation of CSF showed a persistent positive result for oligoclonal bands. The cell count, IgG index and protein levels were now normal. A diagnosis of relapsing-remitting multiple sclerosis was given based on 2017 McDonald Criteria.

The first neurological examination in our neurology department revealed the following: spastic paraparesis with a score of $4 / 5$ (on the MRC - Modified Research Council scale), bilateral Babinski reflex, central facial palsy on the right, lower limbs paresthesia and diminished exteroceptive and mioartrokinetic sensitivity, a relatively normal MMSE (Mini Mental State Examination) score of 29/30. The EDSS (Expanded Disability Status Scale) score on admission was 3.5 .

The investigations performed in our department excluded other autoimmune and infectious diseases and sarcoidosis. The only serological abnormality was positive ANA titers 1:160. ELISA and Western Blot analysis for B. burgdorferi antibodies were repeated with negative results for both $\mathrm{IgG}$ and IgM. The rheumatological evaluation excluded a systemic autoimmune disease.

Given the progression of the neurological signs and symptoms and the evolution of the demyelinating lesions on MRI scans, Teriflunomide therapy (14 mg per day) was initiated.

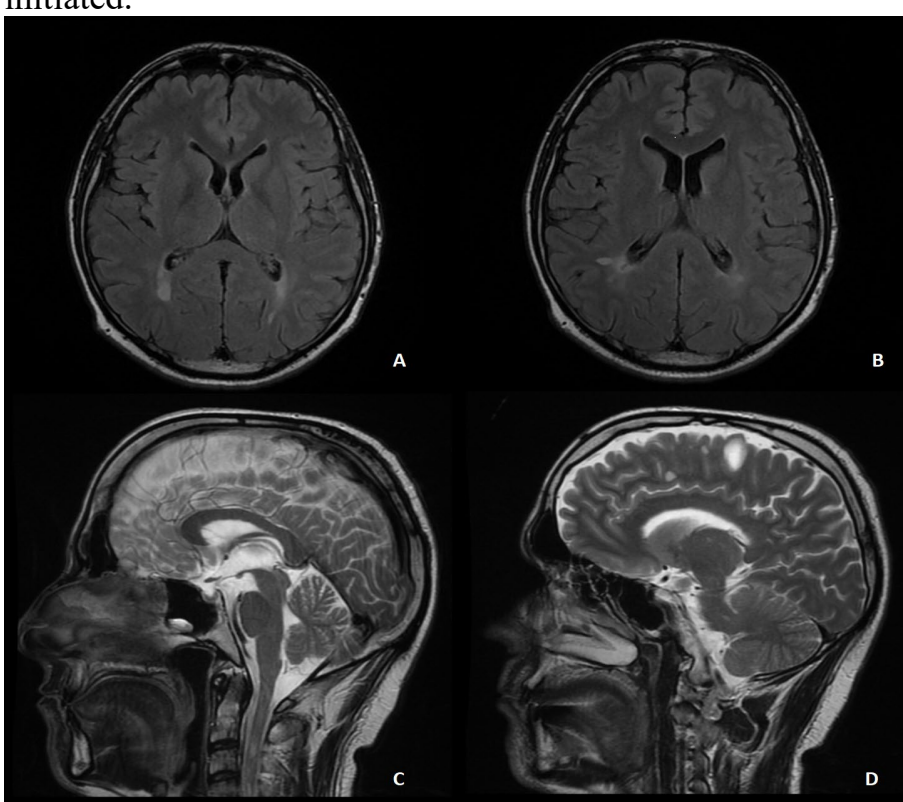

Figure 1. A-B) Axial cross-section MRI images of the brain on FLAIR sequence, showing multiple periventricular lesions $C$-D) Sagittal cross-section MRI images of the brain and cervical spine, T2-weighted, showing short demyelinating lesions in the cervical spine (C2-C3 and C3-C4 level) (C) and multiple subcortical lesions (D). A larger juxtacortical lesion in the right frontal lobe can be observed (D).

During the following year the symptoms did not subside and the patient had another relapse that manifested as paresthesia end exteroceptive hypoesthesia in both hands. Contrast MRI scan of the brain and cervical spine was repeated which showed two new lesions involving the periventricular region adjacent to the right occipital horn, and the corpus callosum, with no signs of activity (Figure 2). 
The EDSS score progressed to 4 points. Teriflunomide therapy was stopped and Interferon beta-1b (250 mcg every other day) was started.

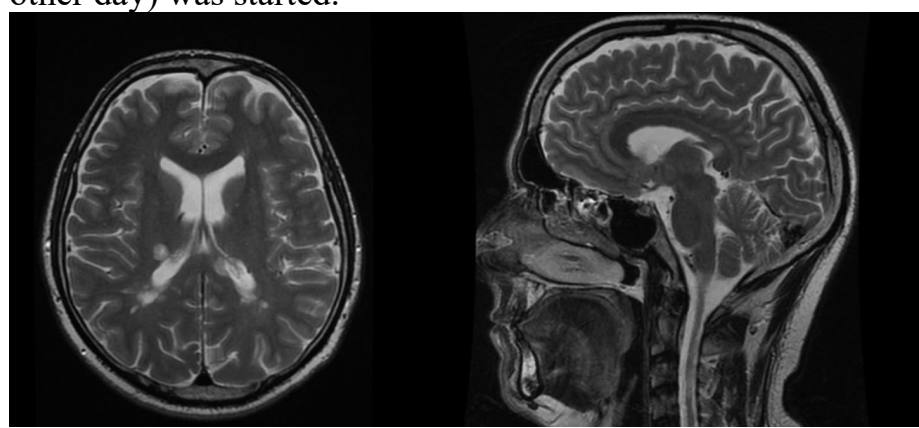

Figure 2. Axial (left) and sagittal (right) cross section MRI images of the brain on FLAIR sequences, revealing periventricular (left) and callosal (right) lesions.

The patient followed the treatment for two months, during which time he presented side effects (injection site reactions with painful, erythematous papules) and peripheral edema in the lower limbs after each injection. After two months he was also admitted for another relapse with tetraparesis (with a score of $4 / 5$ on the MRC scale in all four limbs), marked gait difficulties (ambulatory for 200 meters without aid/rest), paresthesia with exteroceptive hypoesthesia in all four limbs (proximally and distally). The EDSS score was 5.0 points. The MRI scan showed multiple new contrast enhancing lesions in the cervical spine (C1-C2 level and C4-C5 level) (Figure 3 ). The iv pulse methylprednisolone therapy slightly improved the neurological status.

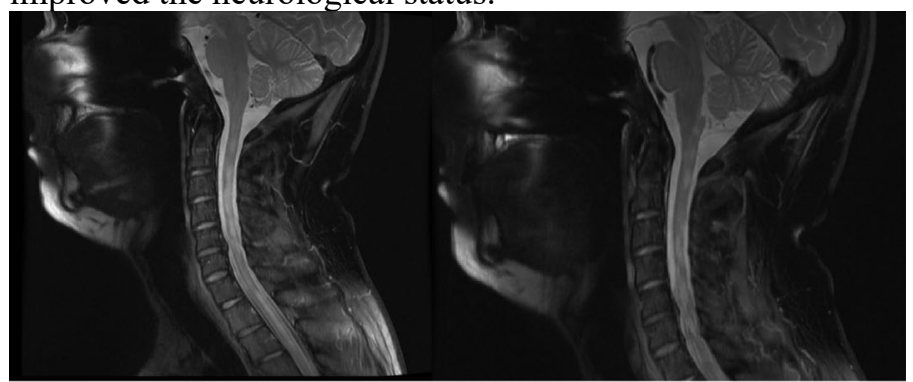

Figure 3. Sagittal cross section MRI images of the brain and cervical spine, PD-weighted, revealing multiple cervical spine lesions.

Due to medication side effects, Interferon 1-b1 was therefore stopped. The high frequency of relapses, with residual disability and the occurrence of new active lesions led to the emergent treatment reconsideration. The patient was proposed for Natalizumab iv (300 $\mathrm{mg}$ once per month). The patient also began a rehabilitation program that included physical exercise and therapeutic massage. Nine months into the treatment, the patient is free of relapses, independent in the daily activities, with an EDSS score of 3.5.

\section{Discussions}

Both the diagnosis and therapeutic management of multiple sclerosis can sometimes prove difficult and require a multidisciplinary approach.

The differential diagnosis of MS includes other idiopathic inflammatory demyelinating disorders (Acute Disseminated
Encephalomyelitis, Schilders's Disease, Balo's Concentric Sclerosis, Neuromyelitis Optica Spectrum Disorders), idiopathic inflammatory non-demyelinating diseases (NeuroBehçet's Disease, Systemic lupus erythematosus, Sjogren Syndrome and Paraneoplastic Neurological Syndromes), sarcoidosis, infectious diseases (Lyme Disease, HTLV-1 associated myelitis, HIV associated CNS involvement), neurosyphilis, metabolic disorders (subacute combined degeneration), CNS vasculitis, Susac's syndrome, toxoplasmosis (15).

Many of the pathologies enumerated could easily be excluded through clinical examination, biological findings and imaging studies. The two most important differential diagnosis for our case were Lyme Disease and idiopathic inflammatory non-demyelinating diseases.

Lyme disease is a chronic infection caused by the neurotropic spirochete Borrelia burgdorferi sensu lato, a bacterium transmitted through tick bites. The nervous system involvement occurs in the $3^{\text {rd }}$ stage (after 6 months of infection). If untreated in the first stage, CNS involvement is almost certain. Commonly, there is a lymphocytic meningitis and multifocal peripheral nerve involvement. Rarely, a clinical picture resembling demyelinating disease may occur along with MRI lesions of vasculitic involvement and/or diffuse CNS demyelination. Detection of bacterial antibodies in CSF is diagnostic for Lyme neuroborreliosis (15). No consensual criteria exist to diagnose neuroborreliosis. In Blanc's et al study, only 30 out of 40 patients had a positive intrathecal anti-Borrelia antibody index. Given the absence of a "gold standard" diagnostic test, their team proposed a list of diagnostic criteria for neuroborreliosis: no past history of neuroborreliosis, positive CSF ELISA serology, positive anti-Borrelia antibody index, favorable outcome after specific antibiotic treatment, and no differential diagnosis (16). Our patient only met 1 out of 5 criteria. Hematological, cardiac, peripheral nervous system are also usual findings during the $3^{\text {rd }}$ stage of the Lyme disease, but those were absent in our patient (15). There is almost no question that our patient really had Lyme disease, but given the aspects we presented above, neuroborreliosis is unlikely to be the cause of the symptoms and the lesions presented on the MRI. It should be noted that the relationship between an infection with $B$. burgdorferi and multiple sclerosis remains obscure and in a study by Chmielewska-Badora et al, the authors stated that "a direct provoking of MS by B. burgdorferi does not look probable, but it cannot be excluded that the Lyme disease agent may aggravate the pathogenic processes in the initial stage of MS and thus increase a number of the symptomatic, clinically diagnosed cases". (17)

Cerebral vasculitis as part of a systemic disorder may have various features on MRI imaging, and the clinical and biological features of the disease include: signs of a systemic disease, headaches, elevated acute-phase reactants (ESR, CRP), inflammatory microcytic anemia, the presence of auto-antibodies (18). None of these changes, were found in our patient with the exception of ANA antibodies. Antineutrophil cytoplasmic antibodies (p-ANCA, c-ANCA), 
rheumatoid factor (RF), circulating immune complexes (CIC) were all within normal range. Tourubah et al also showed that positive titers of antinuclear antibodies occur in $26 \%$ of the patients with multiple sclerosis (19). It should also be noted that Lyme disease could interfere with ANA test inducing a false positive test (20). These could be the explanation for our patients ANA positive autoantibodies.

Primary angiitis of the central nervous system (PACNS) represents a rare inflammatory disease affecting the brain and spinal cord in which systemic involvement is usually absent. The major symptoms of these disease include headaches $(60 \%$ of cases), cognitive impairment $(50 \%$ of cases), neurological focal signs and should be suspected in younger patients. Biological changes described in cases of systemic vasculitis are often absent in PACNS. On MRI, the lesions are typically multifocal, bilateral, hyperintense on T2WI and FLAIR sequences, and can be found in the superficial and deep white matter, and in the cortical gray matter and basal ganglia. A hallmark of PACNS are bilateral stenosis and dilation of blood vessels (21). CSF analysis can reveal abnormal inflammatory findings in $80-90 \%$ of patients with mild lymphocytic pleocytosis and elevated protein level. Oligoclonal bands and immunoglobulin IgG synthesis are occasionally detectable. Nevertheless, the gold standard for diagnosing or excluding PACNS is represented by cerebral biopsy (21).

The armamentarium of approved disease-modifying drugs include the moderately effective, injectable drugs (interferon beta and glatiramer acetate), oral drugs (fingolimod, teriflunomide, dimethyl fumarate), monoclonal antibodies (natalizumab, alemtuzumab, daclizumab, ocrelizumab, rituximab) and immunosuppressive agents (mitoxantrone). (22)

Our patient received Teriflunomide, Interferon beta- $1 \mathrm{~b}$ and Natalizumab sequentially. The first two were stopped due ineffectiveness and adverse effects.

Therapies that have been traditionally considered 'first-line therapies' including the interferons and glatiramer acetate are only partially effective in preventing disease progression in MS and are considered escalation therapies. In clinical practice, these agents are often positioned first line, due to their well-documented safety profile, not because of superior efficacy (23).

The efficacy of natalizumab for treating relapsing-remitting MS was shown in two phase-III trials: the AFFIRM and SENTINEL studies (24), which showed that natalizumab can reduce the annualized relapse rate (ARR) by $68 \%$ and $55 \%$ respectively, in comparison to teriflunomide which reduced the ARR by $31-36 \%$ (25). The AFFIRM and SENTINEL studies also showed that natalizumab lowered the risk of sustained disability progression at 2 years by $42 \%$ and $24 \%$ respectively. (24) A post-hoc analysis of the AFFIRM trial demonstrated that $64 \%$ of patients on natalizumab were free of clinical disease activity, $58 \%$ were free of radiographic disease activity and $37 \%$ were free of combined clinical and radiographic activity throughout the two-year study. (26)
Non-drug-based treatment in MS includes physical therapy (physiotherapy, ergotherapy, logopedics, rehabilitation), sociotherapy and psychotherapy. Impairment of MS patients like spasticity or paresis is primarily a consequence of disease progress (morphological changes), but it can be aggravated by reduced physical activity. Several lines of evidence suggest that MS patients benefit from regular physical activity and exercise with respect to clinical, imaging and physiological parameters. However, the quality of so far realized clinical trials on exercise training in MS do not always satisfy the requirements of a high standard study (27). The lack of evidence suporting exercise-induced neuroplasticity in MS is likely a product of a very small number of papers that do not sufficiently examine the mechanism of action and, therefore, future research might study and reveal the benefits of specifically designed exercise prescriptions to induce functional changes among these patients (28).

\section{Conclusions}

Multiple sclerosis can be a challenging diagnostic because of the perplexing symptoms, the proteiform clinical picture it presents and the large number of illnesses that can cause similar clinical and imaging aspects. Even after a diagnosis is established the clinician's work is not done and finding the optimal treatment for each patient may prove harsher.

The particularity of our case resides in the fact that the patient had an overlap between Lyme disease and multiple sclerosis which lead to confusion and delayed the diagnosis. The patient also had an aggressive form of relapsingremitting multiple sclerosis which did not respond to Interferon beta-1b and Teriflunomide therapy, an induction therapy with Natalizumab should have been started earlier taking into account the prognostic factors in this case.

As new pathophysiological mechanisms are revealed, other tests will become available in the years to come and also novel therapeutics will specifically target these mechanisms in order to speed up the diagnostic process and offer an individualized treatment.

\section{Informed consent}

An informed consent was obtained from the patient participating in the study.

\section{Declaration of conflict of interests}

The authors declare that there was no conflict of interest regarding the publication of this paper.

\section{Author contributions.}

1. Adrian Mihai Cordoș: resident doctor, conception and design of the case report, drafting the discussions section

2. Ioana Simina Barac: resident doctor, drafting the introduction section and comparing the case report to the current literature

3. Dafin Fior Mureșanu: coordinator of the team, given approval of the final version. 


\section{References:}

1. Rostami A, Ciric B. Role of Th17 cells in the pathogenesis of CNS inflammatory demyelination. J Neurol Sci. 2013 Oct 15;333(1-2):76-87.

2. Du J, Wang X, Tan G, Liang Z, Zhang Z, Yu H. The association between genetic polymorphisms of interleukin 23 receptor gene and the risk of rheumatoid arthritis: An updated meta-analysis. Clin Immunol. 2020;210:108250.

3. Grigoriadis N, Pesch V van. A basic overview of multiple sclerosis immunopathology. European Journal of Neurology. 2015;22(S2):3-13.

4. Tarlinton RE, Khaibullin T, Granatov E, Martynova E, Rizvanov A, Khaiboullina S. The interaction between viral and environmental risk factors in the pathogenesis of multiple sclerosis. Int J Mol Sci. 2019 Jan 14;20(2).

5. Silva BA, Leal MC, Farías MI, Avalos JC, Besada $\mathrm{CH}$, Pitossi FJ, et al. A new focal model resembling features of cortical pathology of the progressive forms of multiple sclerosis: Influence of innate immunity. Brain, Behavior, and Immunity. 2018 Mar;69:515-31.

6. Oksenberg JR, McCauley JL. Genetics of multiple sclerosis. Translational Neuroimmunology in Multiple Sclerosis: From Disease Mechanisms to Clinical Applications. 2016 Aug 3;45-54.

7. Naegele M, Martin R. Chapter 3 - The good and the bad of neuroinflammation in multiple sclerosis. In: Goodin DS, editor. Handbook of Clinical Neurology [Internet]. Elsevier; 2014 [cited 2020 Nov 26]. p. 59-87. (Multiple Sclerosis and Related Disorders; vol. 122). Available from:

http://www.sciencedirect.com/science/article/pii/B978044 4520012000030

8. Engelhardt B, Carare RO, Bechmann I, Flügel A, Laman JD, Weller RO. Vascular, glial, and lymphatic immune gateways of the central nervous system. Acta Neuropathol. 2016 Sep 1;132(3):317-38.

9. Kipnis J. Multifaceted interactions between adaptive immunity and the central nervous system. Science. 2016 Aug 19;353(6301):766-71.

10. Văcăraş V, Major ZZ, Buzoianu AD. Brain-derived neurotrophic factor levels under chronic natalizumab treatment in multiple sclerosis. A preliminary report. Neurol Neurochir Pol. 2017 Jun;51(3):221-6.

11. Vacaras V, Major ZZ, Muresanu DF, Krausz TL, Marginean I, Buzoianu DA. Effect of glatiramer acetate on peripheral blood brain-derived neurotrophic factor and phosphorylated TrkB levels in relapsing-remitting multiple sclerosis. CNS Neurol Disord Drug Targets. 2014;13(4):647-51.

12. Miller DH, Weinshenker BG, Filippi M, Banwell BL, Cohen JA, Freedman MS, et al. Differential diagnosis of suspected multiple sclerosis: a consensus approach. Mult Scler. 2008 Nov;14(9):1157-74.

13. Polman null, Uitdehaag null. Drug treatment of multiple sclerosis. West J Med. 2000 Dec;173(6):398-402.

14. Kaunzner UW, Kumar G, Askin G, Gauthier SA, Nealon NN, Vartanian T, et al. A study of patients with aggressive multiple sclerosis at disease onset. Neuropsychiatr Dis Treat. 2016;12:1907-12.

15. Ömerhoca S, Akkaş SY, İçen NK. Multiple sclerosis: diagnosis and differential diagnosis. Noro Psikiyatr Ars. 2018;55(Suppl 1):S1-9.

16. Blanc F, Jaulhac B, Fleury M, de Seze J, de Martino SJ, Remy $\mathrm{V}$, et al. Relevance of the antibody index to diagnose Lyme neuroborreliosis among seropositive patients. Neurology. 2007 Sep 4;69(10):953-8.

17. Chmielewska-Badora J, Cisak E, Dutkiewicz J. Lyme borreliosis and multiple sclerosis: any connection? A seroepidemic study. Ann Agric Environ Med. 2003 Dec 5;7(2):141-3.

18. Berlit P, Kraemer M. Cerebral vasculitis in adults: what are the steps in order to establish the diagnosis? Red flags and pitfalls. Clin Exp Immunol. 2014 Mar;175(3):419-24.

19. Tourbah A, Clapin A, Gout O, Fontaine B, Liblau R, Batteux F, et al. Systemic autoimmune features and multiple sclerosis: a 5-year follow-up study. Arch Neurol. 1998 Apr;55(4):517-21.

20. Lovece S, Stern R, Kagen LJ. Effects of rheumatoid factor, antinuclear antibodies and plasma reagin on the serologic assay for Lyme disease. J Rheumatol. 1991 Dec;18(12):1813-8.

21. Beuker C, Schmidt A, Strunk D, Sporns PB, Wiendl H, Meuth SG, et al. Primary angiitis of the central nervous system: diagnosis and treatment. Ther Adv Neurol Disord. 2018;11:1756286418785071.

22. Tanasescu R, Ionete C, Chou I-J, Constantinescu CS. Advances in the treatment of relapsing-remitting multiple sclerosis. Biomed J. 2014 Apr;37(2):41-9.

23. Nicholas JA, Racke MK, Imitola J, Boster AL. First-line natalizumab in multiple sclerosis: rationale, patient selection, benefits and risks. Ther Adv Chronic Dis. 2014 Mar;5(2):62-8.

24. Polman CH, O’Connor PW, Havrdova E, Hutchinson M, Kappos L, Miller DH, et al. A randomized, placebocontrolled trial of natalizumab for relapsing multiple sclerosis. N Engl J Med. 2006 Mar 2;354(9):899-910.

25. Rommer PS, Milo R, Han MH, Satyanarayan S, Sellner J, Hauer L, et al. Immunological aspects of approved ms therapeutics. Front Immunol [Internet]. 2019 Jul 11 [cited 2020 Nov 26];10. Available from: https:/www.ncbi.nlm.nih.gov/pmc/articles/PMC6637731/

26. Havrdova E, Galetta S, Hutchinson M, Stefoski D, Bates $\mathrm{D}$, Polman $\mathrm{CH}$, et al. Effect of natalizumab on clinical and radiological disease activity in multiple sclerosis: a retrospective analysis of the Natalizumab Safety and Efficacy in Relapsing-Remitting Multiple Sclerosis (Affirm) study. Lancet Neurol. 2009 Mar;8(3):254-60.

27. Döring A, Pfueller CF, Paul F, Dörr J. Exercise in multiple sclerosis -- an integral component of disease management. EPMA J. 2011 Dec 24;3(1):2

28. Sandroff BM, Jones CD, Baird JF, Motl RW. Systematic review on exercise training as a neuroplasticity-inducing behavior in multiple sclerosis. Neurorehabil Neural Repair. 2020;34(7):575-88. 